\title{
Retrospective Analysis of Bacterial Isolates during Blood Stream Infections in Children with Chemotherapy-induced Febrile Neutropenia: A Single Centre Experience
}

\author{
Abhilasha Sampagar ${ }^{1}$ \\ Prathamesh Pawashe ${ }^{2}$ \\ B. R. Ritesh ${ }^{1}$ \\ Dubey Shiv ${ }^{2}$ \\ ${ }^{1}$ Department of Paediatrics, KAHER's Jawaharlal Nehru Medical \\ College, Belagavi, Karnataka, India \\ 2 Department of Pediatrics, House Surgeon, KAHER's Jawaharlal \\ Nehru Medical College, Belagavi, Karnataka, India \\ ${ }^{3}$ Department of Urology, KAHER's Jawaharlal Nehru Medical College, \\ Belagavi, Karnataka, India
}

\author{
Shridhar C. Ghagne ${ }^{3}$ Neha Patil ${ }^{2}$
}

\begin{abstract}
Address for correspondence Abhilasha Sampagar, MD, FPHO, Department of Paediatrics, KAHER's Jawaharlal Nehru Medical College, Belagavi, Karnataka, 590010, India (e-mail: abhilasha.pedia@gmail.com).
\end{abstract}

Ind J Med Paediatr Oncol 2021;42:540-546.

\begin{abstract}
Keywords

- febrile neutropenia

- antibiotics

- cancer

- bloodstream infections

- antibiotic resistance
\end{abstract}

Introduction The recent advances in cancer treatment have resulted in significant improvement in the outcome of pediatric cancers. However, febrile neutropenia (FN) is the most important cause of mortality and morbidity in pediatric cancer patients and is a crucial limiting factor for the outcome. The greatest threat that we are facing is the emergence of pan drug-resistant (PDR) organisms.

Objectives To study bacterial organisms causing bloodstream infections (BSI) during febrile neutropenia episodes, their antibiotic sensitivity pattern, impact on treatment outcome during the intensive phase of chemotherapy, and the association between prior administration of antibiotics and emergence of multidrug-resistant organisms (MDR).

Materials and Methods This retrospective study was conducted in patients between the age group of 0 to 18 years who were treated for malignancies in the division of pediatric oncology at a tertiary center from August 2017 to December 2020. Blood cultures were collected under aseptic precautions, and they were processed as per the Clinical and Laboratory Standard Institute Guideline (CLSI) 2017.

Results A total of 122/159 (76.7\%) patients were diagnosed to have hematological malignancies, and $37 / 159$ (23.3\%) patients were found to be suffering from solid tumors. A total of 309 episodes of FN were documented and 386 cultures were sent, out of which $87 / 386(22.53 \%)$ cultures were positive for bacteria and 2/386 (2.2\%) for fungi. Gram-negative isolates were seen in 51/87 (58.62\%) cultures and Gram-positive in 36/87 (41.37\%) cultures. Burkholderia cepacia and coagulase-negative Staphylococci (CONS) were the commonest found Gram-negative and Gram-positive bacteria,
DOI https://doi.org/ 10.1055/s-0041-1740313. ISSN 0971-5851.

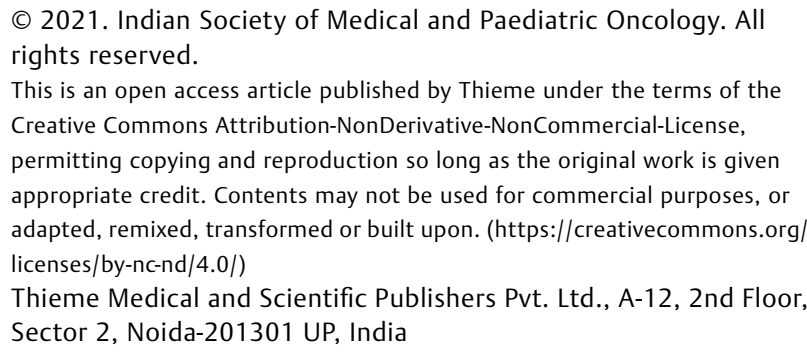

(C) 2021. Indian Society of Medical and Paediatric Oncology. All rights reserved.

This is an open access article published by Thieme under the terms of the Creative Commons Attribution-NonDerivative-NonCommercial-License, permitting copying and reproduction so long as the original work is given appropriate credit. Contents may not be used for commercial purposes, or adapted, remixed, transformed or built upon. (https://creativecommons.org/ licenses/by-nc-nd/4.0/)

Thieme Medical and Scientific Publishers Pvt. Ltd., A-12, 2nd Floor, Sector 2, Noida-201301 UP, India 
respectively. MDR bacterial strains were seen in 44/87 (50.57\%) cultures and PDR strains in $8 / 87$ (9.2\%) cultures. Resistance was higher with Klebsiella species and CONS. There were six mortalities during the induction phase of acute leukemia treatment, out of which $4 / 6(66.66 \%)$ were due to MDR infections, $1 / 6(16.6 \%)$ due to fungal infection and chemotherapy refractoriness each.

Conclusion Proven bacterial infections were determined in $22.53 \%$ of febrile neutropenia episodes. Most BSI in patients with febrile neutropenia were caused by Gramnegative bacteria. Indiscriminate use of higher antibiotics before referral led to the emergence of MDR organisms, thus compromising the outcome. Our study emphasizes the fact that antibiotic stewardship is a crucial task to counter MDR bacteremiarelated morbidity and mortality in neutropenic children.

\section{Introduction}

The recent advances in cancer treatment have resulted in significant improvement in the outcome of pediatric cancers. ${ }^{1}$ However, febrile neutropenia (FN) and the emergence of multidrug-resistant (MDR) organisms remain one of the important limiting factors. ${ }^{2} \mathrm{FN}$ is the most common complication of therapy seen in the pediatric age group with cancer and is one of the major causes of morbidity and mortality in children and young adults treated for cancer. ${ }^{2,3}$ The chances of infection are higher in these patients due to immunosuppression, which is caused by the disease itself and also due to strong chemotherapeutic agents. ${ }^{4}$ Moreover, exposure to pathogens is increased due to the usage of indwelling venous catheters, chemo ports, and frequent insertion of urinary catheters. ${ }^{5}$ The mortality rate associated with febrile neutropenia in children is higher in hematological malignancies compared to those with solid tumors. ${ }^{6}$ Apart from infectionrelated mortality and morbidities, other consequences of FN can include dose reductions of chemotherapeutic drugs and treatment delays, which can have a substantial impact on the curative potential of treatment. ${ }^{7}$ Organisms causing neutropenia in pediatric cancer patients show an increased rate of Gram-negative infections including Burkholderia cepacia, Pseudomonas aeruginosa, Acinetobacter species, Stenotrophomonas maltophilia, Escherichia coli, and Klebsiella species and change in their sensitivity patterns. ${ }^{8-10}$ The Gram-positive bacteria are common in the setting of use of central venous catheters and mucositis. Fungi such as Candida species or Aspergillus species are more likely encountered after prolonged neutropenia and administration of broadspectrum antibiotics. ${ }^{11}$ Development of MDR organisms is one of the major challenges to the success of treatment, which is mainly due to the indiscriminate use of antibiotics. Knowledge of common pathogens and their antibiotic sensitivity patterns is crucial for timely empirical therapy, preventing complications such as septic shock, and thus decreasing mortality. ${ }^{12,13}$

Therefore, the present study aims to determine the common organisms that cause febrile neutropenia and their sensitivity patterns in pediatric cancer patients, the impact of infection on treatment outcome, and the association between prior administration of antibiotics and emergence of multi-drug resistant organisms.

\section{Materials and Methods}

This retrospective study was conducted in children below 18 years of age, who were treated for malignancies in the division of pediatric oncology at a tertiary center between August 2017 and December 2020. The patients who were not in the intensive phase of chemotherapy were excluded from the study. We included 159 eligible patients who met the inclusion criteria during this period. The primary outcome measure was an episode of febrile neutropenia in pediatric cancer patients followed by the secondary outcome measure, which was bloodstream infections with positive bacterial cultures. The clinical criteria for febrile neutropenia were defined as per the European Society of Medical Oncology, that is an oral temperature $338.5^{\circ} \mathrm{C}$ or two consecutive readings of $38^{\circ} \mathrm{C}$ for 2 hours and an absolute neutrophil count of $<0.5 \times 10^{9} / \mathrm{L}$, or expected to fall $<0.5 \times 10^{9} / \mathrm{L}$. BSI was defined as isolation of one or more recognized bacterial pathogens from one or more blood cultures in the presence of fever $\left(>38^{\circ}\right.$ C) and chills or rigors within 24 hours of a positive blood culture being collected. ${ }^{14}$ When mixed isolates were obtained with one being an accepted pathogen, the potential contaminant organism was disregarded.

All blood samples were collected as per the standard ageappropriate sample collection protocol. The clinical data were obtained from the requisition forms and clinical records of the patients. All samples were processed as per the standard microbiology laboratory operating procedures. The bacterial isolates were identified by their colonial morphology, Gram staining, and different biochemical reactions using standard techniques. Criteria for antimicrobial sensitivity testing were used as per the Clinical Laboratory Standard Institute (CLSI). ${ }^{15}$ Antimicrobial sensitivity testing was done on Mueller-Hinton agar (MHA) using the Kirby-Bauer disc diffusion method. Commercially available discs (Hi-Media) were used. Zones of inhibition were measured the next day and were correlated with CLSI interpretive breakpoints to characterize them as sensitive, intermediate, and resistant. For Gram-positive organisms, the antibiotics to be tested and reported were 
542 Blood Stream Infections in Children with Chemotherapy-induced Febrile Neutropenia Sampagar et al.

chosen from the following (depending on the organism isolated): penicillin (10 units), erythromycin $(15 \mu \mathrm{g})$, clindamycin $(2 \mu \mathrm{g})$, gentamicin $(10 \mu \mathrm{g})$, and high level $(30 \mu \mathrm{g})$, amoxicillinclavulanate $(20 / 10 \mu \mathrm{g})$, cefoxitin $(30 \mu \mathrm{g})$, levofloxacin $(5 \mu \mathrm{g})$, vancomycin $(30 \mu \mathrm{g} /$ minimum inhibitory concentration), linezolid $(30 \mu \mathrm{g})$, and cotrimoxazole $(1.25 / 23.75 \mu \mathrm{g})$. For Gramnegative organisms, the antibiotics for respective organisms were chosen from the following: ciprofloxacin $(5 \mu \mathrm{g})$, levofloxacin $(5 \mu \mathrm{g})$, norfloxacin $(5 \mu \mathrm{g})$, gentamicin $(10 \mu \mathrm{g})$, amikacin $(30 \mu \mathrm{g})$, cefpodoxime $(30 \mu \mathrm{g})$, cefotaxime $(30 \mu \mathrm{g})$, ceftazidime $(30 \mu \mathrm{g})$, cefepime $(30 \mu \mathrm{g})$, piperacillin + tazobactum $(100 / 10 \mu \mathrm{g})$, imipenem $(10 \mu \mathrm{g})$, and colistin $(10 \mathrm{mcg})$. MRSA was tested using MHA with a cefoxitin disc $(30 \mu \mathrm{g})$ using the Kirby-Bauer disc diffusion method as per the CLSI guidelines. MIC could be calculated for a few cultures by an automated culture system namely Beckman Coulter's MicroScan Walkaway. Standard definitions were used for the terms ESBL, $\beta$-lactam $\beta$-lactamase inhibitor (BLBLI), and carbapenem resistance. MDR was defined as acquired n-susceptibility to at least one agent in three or more antimicrobial categories, and PDR was defined as non-susceptibility to all agents in all antimicrobial categories.

\section{Statistical Analysis}

The categorical variables are expressed as percentages. The difference between categorical variables was evaluated using the chi-square test. A $p$-value of $<0.05$ was considered significant. In case of any violation of assumptions of chisquare test, simulation was done for chi square test. Data analysis was done using the software SPSS Version-20, USA, and Excel. Categorical variables were given in the form of frequency table.

\section{Ethical Statements}

The procedures followed were in accordance with the ethical standards of our medical college ethical committee and with the Helsinki Declaration of 1964, as revised in 2013. Due to the retrospective nature of this study, a waiver of consent was approved by the Jawaharlal Nehru Medical College Ethics Committee. The reference number is MDC/DOME/ 156 and was approved on 1/10/2017.

\section{Results}

Over a duration of 3 years, 159 children between the age of 0 and 18 years, with different malignancies presented to our center. The mean age was 5.2 years (range: $0.8-17.2$ years) and the male to female ratio was 1.35:1. The mean duration from the onset of symptoms to referral to our oncology center was 38.7 days. Acute lymphoblastic leukemia (ALL) was the most common 84/159 (52.8\%) malignancy. Acute myeloid leukemia (AML) constituted 21/159 (13.2\%), lymphomas constituted 17/159 (10.7\%), out of which 9 were Hodgkin and 8 were non-Hodgkin. Solid tumors were 37/159 (23.3\%), consisting of renal tumors $(8 / 37$ [21.6\%]), neuroblastoma (8/37 [21.6\%]), Ewing sarcoma $7 / 37$ [18.9\%]), germ cell tumors (6/37 [16.2\%]), and rhabdomyosarcoma (5/37 [13.5\%]). There were $3 / 37(8.1 \%)$ rare tumors.
Table 1 Overall distribution of the organisms isolated in the blood cultures

\begin{tabular}{|c|c|}
\hline \multicolumn{2}{|l|}{ Total isolates $=89$} \\
\hline Gram-positive bacteria & $36(41.37 \%)$ \\
\hline Gram-negative bacteria & $51(58.62 \%)$ \\
\hline Fungus & $2(2.17 \%)$ \\
\hline
\end{tabular}

Among these patients, 309 episodes of febrile neutropenia were documented and 386 cultures were sent during episodes of febrile neutropenia. The median absolute neutrophil count was 210 (range: $0-560$ ). The median duration of neutropenia was 8 (range: 1-32.5) days. In addition, $87 / 386(22.53 \%)$ cultures were positive for bacteria and $2 / 386(2.2 \%)$ for fungus.

- Table 1 shows the overall distribution of the isolates, with 36/87 (41.37\%; 95\% confidence interval [CI]: 34\%-50\%) being Gram-positive bacteria and 51/87 (58.62\%; 95\%CI: $52-$ $65 \%)$ were Gram-negative bacteria. The most common bacterial isolates from blood are depicted in - Table 2 with $B$. cepacia 14/51 (27.45\%) being the most common among Gram-negative bacteria and coagulase-negative Staphylococci 32/36 (88.88\%) among Gram-positive bacteria. Among the Gram-negative bacteria, 14/51 (27.45\%) were B. cepacia, 10/51 (19.60\%) were Acinetobacter species, 8/51 (15.68\%) were Enterobacter species, 10/51 (19.60\%) were Klebsiella species, and 4/51 (7.84\%) were P. aeruginosa and E. coli each. Among the Gram-positive bacteria, 32/36 (88.88\%) were CONS including Staphylococcus hemolyticus and S. epidermidis, and $1 / 36(8.32 \%)$ were S. aureus. MDR strains were seen in 44/87 (50.57\%; 95\%CI: 44-56\%) cultures, while PDR strains were seen in $8 / 87(9.2 \%)$ cultures (-Tables 3 and $\mathbf{4}$ ). The maximum antibiotic resistance was seen in Klebsiella species and CONS. Extended-spectrum beta-lactamase (ESBL)

Table 2 Most common bacterial isolates in blood

\begin{tabular}{|l|l|}
\hline Organisms & $\boldsymbol{n}(\%)$ \\
\hline Gram-negative isolates in blood $(\boldsymbol{n}=\mathbf{5 1})$ & \multicolumn{2}{|l|}{} \\
\hline Pseudomonas aeruginosa & $4(7.84)$ \\
\hline Acinetobacter species & $10(19.60)$ \\
\hline Klebsiella species & $10(19.60)$ \\
\hline Enterobacter species & $8(15.68)$ \\
\hline Escherichia coli & $4(7.84)$ \\
\hline Serratia marcescens & $1(1.96)$ \\
\hline Burkholderia cepacia & $14(27.45)$ \\
\hline Gram-positive isolates in blood (n=36) & \\
\hline Staphylococcus epidermidis & $6(16.66)$ \\
\hline Staphylococcus aureus & $1(2.77)$ \\
\hline Methicillin-resistant Staphylococcus aureus & $2(5.55)$ \\
\hline Enterococcus faecalis & $1(2.77)$ \\
\hline Staphylococcus haemolyticus & $6(16.66)$ \\
\hline Other coagulase-negative Staphylococci & $20(55.55)$ \\
\hline
\end{tabular}


Table 3 Antibiotic sensitivity patterns of Gram-positive isolates

\begin{tabular}{|l|l|l|l|l|l|l|l|l|l|l|}
\hline Organism & SXT & VA & C & LNZ & TEC & CLR & CIP & NET & TE & R \\
\hline $\begin{array}{l}\text { Staphylococcus } \\
\text { epidermidis } \\
(n=6)\end{array}$ & $3(50 \%)$ & $5(83.3 \%)$ & $5(83.3 \%)$ & $4(66.6 \%)$ & $4(66.6 \%)$ & Not done & Not done & $3(50 \%)$ & $1(16.6 \%)$ & $3(50 \%)$ \\
\hline $\begin{array}{l}\text { MRSA } \\
(n=2)\end{array}$ & Not done & $1(50 \%)$ & Not done & $1(50 \%)$ & $1(50 \%)$ & Not done & Not done & Not done & $1(50 \%)$ & $2(100 \%)$ \\
\hline $\begin{array}{l}\text { Enterococcus } \\
(n=1)\end{array}$ & $0(0 \%)$ & $1(100 \%)$ & $1(100 \%)$ & $1(100 \%)$ & Not done & Not done & Not done & Not done & $1(100 \%)$ & Not done \\
\hline $\begin{array}{l}\text { Staphylococcus } \\
\text { haemolyticus } \\
(n=6)\end{array}$ & Not done & $3(50 \%)$ & $2(33.3 \%)$ & $4(66.6 \%)$ & $2(33.3 \%)$ & Not done & Not done & $2(33.3 \%)$ & $5(83.3 \%)$ & $1(16.6 \%)$ \\
\hline $\begin{array}{l}\text { CONS } \\
(n=20)\end{array}$ & $9(45 \%)$ & $12(60 \%)$ & $14(70 \%)$ & $13(65 \%)$ & $9(45 \%)$ & $10(50 \%)$ & $15(75 \%)$ & $14(70 \%)$ & $10(20 \%)$ & $12(60 \%)$ \\
\hline $\begin{array}{l}\text { Staphylococcus } \\
\text { aureus } \\
\text { (methicillin- } \\
\begin{array}{l}\text { sensitive) } \\
(n=1)\end{array}\end{array}$ & Not done & $1(100 \%)$ & Not done & $1(100 \%)$ & $1(100 \%)$ & Not done & Not done & Not done & $1(100 \%)$ & Not done \\
\hline
\end{tabular}

CONS, coagulase-negative Staphylococci; C, chloramphenicol; CIP, ciprofloxacin; CLR, clarithromycin; LNZ, linezolid; NET, netilmicin; R, rifampicin; SXT, sulfamethoxazole/trimethoprim; TE, tetracycline; TEC, teicoplanin; VA, vancomycin.

Table 4 Antibiotic sensitivity patterns of gram-negative isolates

\begin{tabular}{|c|c|c|c|c|c|c|c|c|c|c|c|c|}
\hline Organism & AK & CS & TGC & MRP & SXT & CTX & IMI & GEN & LEV & CAZ & CIP & TZP \\
\hline $\begin{array}{l}\text { Pseudomonas } \\
(n=4)\end{array}$ & $3(75 \%)$ & $3(75 \%)$ & - & $2(50 \%)$ & - & - & $1(25 \%)$ & $1(25 \%)$ & $2(50 \%)$ & $2(50 \%)$ & $2(50 \%)$ & 1 (25\%) \\
\hline $\begin{array}{l}\text { Acinetobacter } \\
(n=10)\end{array}$ & $6(60 \%)$ & $1(10 \%)$ & $2(20 \%)$ & 7 (70\%) & $6(60 \%)$ & $6(60 \%)$ & $1(10 \%)$ & $5(50 \%)$ & $5(50 \%)$ & $4(40 \%)$ & $4(40 \%)$ & $2(20 \%)$ \\
\hline $\begin{array}{l}\text { Klebsiella } \\
(n=10)\end{array}$ & $5(50 \%)$ & $6(60 \%)$ & $8(80 \%)$ & $5(50 \%)$ & $4(40 \%)$ & $0(0 \%)$ & 7 (70\%) & $6(60 \%)$ & $4(40 \%)$ & $1(10 \%)$ & $3(30 \%)$ & $5(50 \%)$ \\
\hline $\begin{array}{l}\text { Burkholderia } \\
(n=14)\end{array}$ & $0(0 \%)$ & $0(0 \%)$ & $0(0 \%)$ & $3(21.4 \%)$ & 10 (71.4\%) & Not done & Not done & $0(0 \%)$ & 12 (85.7\%) & 1 (7.1\%) & Not done & $0(0 \%)$ \\
\hline $\begin{array}{l}\text { Enterobacter } \\
(n=8)\end{array}$ & $6(75 \%)$ & $3(37.5 \%)$ & $4(50 \%)$ & $4(50 \%)$ & 1 (12.5\%) & $1(12.5 \%)$ & 5 (62.5\%) & $2(25 \%)$ & $5(62.5 \%)$ & $2(25 \%)$ & $3(37.5 \%)$ & $3(37.5 \%)$ \\
\hline $\begin{array}{l}\text { E. coli } \\
(n=4)\end{array}$ & 1 (25\%) & $2(50 \%)$ & $3(75 \%)$ & $2(50 \%)$ & Not done & $0(0 \%)$ & 1 (25\%) & $2(50 \%)$ & $2(50 \%)$ & $0((0 \%)$ & $1(25 \%)$ & 1 (25\%) \\
\hline $\begin{array}{l}\text { Serratia } \\
(n=1)\end{array}$ & 1 (100\%) & $0(0 \%)$ & 1 (100\%) & 1 (100\%) & 1 (100\%) & $0(0 \%)$ & $1(100 \%)$ & $1(100 \%)$ & 1 (100\%) & $0(0 \%)$ & 1 (100\%) & 1 (100\%) \\
\hline
\end{tabular}

Abbreviations: AK, amikacin; CAZ, ceftazidime; CIP, ciprofloxacin; CS, colistin; CTX, cefotaxime; GEN, gentamicin; IMI, imipenem; LEV, levofloxacin; MRP, meropenem; SXT, sulfamethoxazole/trimethoprim; TCC, tigecycline; TZP, piperacillin/tazobactam.

sensitivity was done for E. coli, Klebsiella, and Enterobacter species. A higher proportion of ESBL isolates were seen in Klebsiella species, 9/10 (90\%) followed by E. coli $2 / 4(50 \%)$, and Enterobacter species (2/8 [25\%]). Only one culture grew Serratia marcescens, which was also an ESBL-producing organism. Most of the MDR Gram-negative organisms were sensitive to colistin 18/40, (45\%; 95\%CI: $34-54 \%)$ and MDR Gram-positive organisms 3/4, (75\%; 95\%CI: 68-80\%) were sensitive to vancomycin. Bacterial infection was the leading cause of mortality during the intensive phase of treatment. There were six mortalities during the induction phase of treatment in acute leukemias, out of which 3/6 (50\%) were due to PDR bacterial infections, $1 / 6$ (16.6\%) due to MDR bacterial infection, and $1 / 6(16.6 \%)$ due to invasive aspergillosis and chemotherapy refractoriness each. In addition, 3/6 (50\%) patients succumbed to Gram-negative septi- cemia, who were PDR (1 B. cepacia, 1 Klebsiella species, and 1 Enterobacter species) and 1 to Gram-positive (MRSA, which was MDR). There were no mortalities in solid tumor cases during the intensive phase of treatment. Another interesting finding of our study was that 39/52 (75\%; 95\%CI: 70-80\%) patients, who harbored MDR or PDR bugs, were pre-treated with broad-spectrum antibiotics before being referred to our

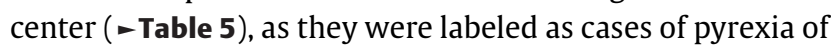
unknown origin. This was statistically significant $(p<0.05)$ when compared with only $13 / 52$ (25\%) patients without pretreatment with antibiotics who grew MDR or PDR bacteria. In these children, the mean duration of administration of oral antibiotics was 6.2 days and that of intravenous antibiotics was 9.6 days.

The two fungal isolates were Candida glabrata. Their sensitivity pattern could not be done but they 
Table 5 Association between prior administration of antibiotic and emergence of MDR/PDR bugs $(p<0.05)$

\begin{tabular}{|l|l|l|l|}
\hline Sl. no. & $\begin{array}{l}\text { Treatment regime of patients who harbored MDR or } \\
\text { PDR organisms }(\boldsymbol{n}=\mathbf{5 2})\end{array}$ & $\boldsymbol{n}(\%)$ & $\boldsymbol{p}$-Value \\
\hline $\mathbf{1}$ & Patients not pre-treated with antibiotics & $13(25 \%)$ & $p<0.05$ \\
\hline $\mathbf{2}$ & Pre-treated with broad-spectrum antibiotics & $39(75 \%)$ & $p<0.05$ \\
\hline $\mathbf{2 a}$ & Pre-treated with oral broad-spectrum antibiotics & $10(19.2 \%)$ & \\
\hline $\mathbf{2 b}$ & Pre-treated with intravenous broad-spectrum antibiotics. & $29(55.7 \%)$ & \\
\hline
\end{tabular}

showed good clinical response to voriconazole and amphotericin B.

\section{Discussion}

Cancer patients are often immunocompromised because of the disease process itself and also due to chemotherapyrelated neutropenia. ${ }^{16}$ In addition, there are other associated risk factors for acquiring infection, such as central lines, longterm catheterization, and mucositis due to cytotoxic agents. $^{14,17,18}$ Infectious complications are a serious cause of morbidity and mortality in patients with underlying hematological malignancies. Moreover, in solid tumors, although neutropenia is not for a prolonged duration, the presence of multiple other risk factors are responsible for an immunocompromised state, such as obstruction caused by the tumor, disruption of normal anatomical barriers, therapeutic procedures, radiation, and use of medical devices such as central lines and catheters. ${ }^{19}$

In our study, only five patients with AML had central catheters, out of which three had chemo ports and two had PICC lines whose cultures were positive. Most of the patients were managed with peripheral lines due to financial constraints. Hence, only five patients were found to have catheter-related bloodstream infections. There are various guidelines available at national and international levels regarding the management of febrile neutropenia. ${ }^{20}$ Adoption of these guidelines into an individual oncology unit is posed by challenges such as differences in the predominant pathogens, their sensitivity patterns, and/or healthcare-associated economic conditions. Regardless of that, clinical vigilance and immediate treatment are the universal keys to managing neutropenic patients with fever and/or infection. ${ }^{21}$ The present study was performed to assess the incidence of BSIs in children with cancer and to assess the microbiological profile and the impact of infections on the outcome.

The bacterial culture positivity rate was high at our center (22.53\%) and this was comparable to other Indian studies done by Kapoor et al and Siddaiahgari et al. ${ }^{22,23}$ In our study, Gram-negative bacteremia was more common $(58.62 \%)$ as compared to Gram-positive bacteremia (41.37\%). Similar results were reported by Indian studies and by Kar et al in Turkey. ${ }^{17,22,23}$ However, the data from the western countries reveal a changing trend of predominant Gram-positive organisms and this is probably due to the use of more indwelling catheters. ${ }^{24}$ Among the Gram-negative organ- isms, B. cepacia was the most commonly (27.7\%) isolated organism, followed by Acinetobacter species and Klebsiella species, whereas similar studies on febrile neutropenia patients reported E. coli, Klebsiella species, and P. aeruginosa as the commonest Gram-negative organisms.,22,23 Grampositive bacteria mainly consisted of CONS (55.5\%) followed by $S$. hemolyticus (6\%) and MRSA (2\%). The distribution of Gram-positive organisms was comparable to other Indian and western studies. ${ }^{4,22,23}$ These findings show that immunocompromised patients are colonized by more or less similar organisms in different parts of the world. The predominance of $B$. cepacia and its sensitivity and good response to levofloxacin was a unique finding of our study. A matter of great concern at our center was the emergence of MDR and PDR bacteria. All PDR organisms were Gram-negative and consisted of Klebsiella species (3), Enterobacter species (3), and B. cepacia (2). These organisms (PDR and MDR) accounted for $66.6 \%(4 / 6)$ of mortalities during the induction phase of treatment of leukemia. Although an increasing trend in resistance is being reported globally, the number of resistant strains in our study was significantly higher as compared with other studies. Alarmingly, we found that $90 \%$ of Klebsiella species, 50\% of E. coli, and 25\% of Enterobacter species were ESBL-producing organisms. ${ }^{4,17,24}$ A study done on neutropenic cancer patients in Egypt revealed a resistance rate as high as $68.6 \%{ }^{25} \mathrm{~A}$ probable explanation for the higher number of resistant strains at our center is that most of the cases had a delayed presentation, as our center, being located in the periphery, caters to a predominantly rural population and most of these patients (75\%) were pre-treated with broad-spectrum antibiotics due to alternative diagnoses. ${ }^{26}$ The association between prior administration of antibiotics and the presence of resistant organisms was statistically significant. Most of the MDR Gram-negative organisms were sensitive to colistin. Similar findings were reported in other studies done on neutropenic patients harboring MDR organisms. ${ }^{27,28}$ In our study, most of the MDR Gram-positive organisms were sensitive to vancomycin, whereas Garg et al noted that more than $50 \%$ of MDR Gram-positive organisms were vancomycin resistant. ${ }^{29}$

This can be attributed to the fact that vancomycin was not used for empirical therapy for our patients. To conclude, these results on microbiologic profile and antibiotic sensitivity patterns of the isolates will be beneficial in revising antibiotic protocols and in developing strategies for dealing with the high prevalence of MDR and PDR organisms. Institutional antibiotic policy (in place) is a necessary step toward 
early recovery from febrile neutropenia, with rationale empirical antibiotics usage according to the culture sensitivity pattern. Our study emphasizes the fact that antibiotic stewardship is a crucial task to counter MDR bacteremia-related morbidity and mortality in neutropenic children. The limitations of this study are that it was a retrospective study and the data were obtained from a single pediatric oncology center.

\section{Conclusion}

The proper identification of trends of bacteriological isolates in cancer patients is necessary to develop an appropriate antibiotic policy to combat these infections in a proper way and at an earlier stage. The emerging resistance pattern calls for the judicious use of antibiotics, surveillance of resistant patterns, and an active infection control policy within the oncology unit. MDR and PDR organisms were responsible for most of the mortalities. Every institution should be performing these kinds of studies to establish their own sensitivity patterns and determine their empirical antibiotics of choice on the arrival of the patient to the institute as per the history of the patient regarding the treatment he/she received in the community prior to their presentation to the institute. The indiscriminate use of higher antibiotics before referral led to the emergence of MDR organisms, thus compromising the outcome as $66.6 \%$ (4/6) of mortalities occurred due to MDR infections.

Funding
None.

Conflict of Interest

None declared.

\section{References}

1 Butler E, Ludwig K, Pacenta HL, Klesse LJ, Watt TC, Laetsch TW. Recent progress in the treatment of cancer in children. CA Cancer J Clin 2021;71(04):315-332

2 Lucas AJ, Olin JL, Coleman MD. Management and preventive measures for febrile neutropenia. P\&T 2018;43(04):228-232

3 O'Connor D, Bate J, Wade R, et al. Infection-related mortality in children with acute lymphoblastic leukemia: an analysis of infectious deaths on UKALL2003. Blood 2014;124(07): 1056-1061

4 Rajeswari B, Nair RK, Guruprasad CS, Nair M, Thankamony P, Parukutty K. Infections during induction chemotherapy in children with acute lymphoblastic leukemia-profile and outcomes: experience from a cancer center in South India. Indian J Med Paediatr Oncol 2018;39(02):188-192

5 Oude Nijhuis CS, Daenen SM, Vellenga E, et al. Fever and neutropenia in cancer patients: the diagnostic role of cytokines in risk assessment strategies. Crit Rev Oncol Hematol 2002;44(02): 163-174

6 Jacob LA, Lakshmaiah KC, Govindbabu K, et al. Clinical and microbiological profile of febrile neutropenia in solid tumors and hematological malignancies at a tertiary cancer care center in South India. Indian J Cancer 2014;51(04):464-468

7 Weycker D, Barron R, Kartashov A, Legg J, Lyman GH. Incidence, treatment, and consequences of chemotherapy-induced febrile neutropenia in the inpatient and outpatient settings. J Oncol Pharm Pract 2014;20(03):190-198

8 Sung L, Gamis A, Alonzo TA, et al. Infections and association with different intensity of chemotherapy in children with acute myeloid leukemia. Cancer 2009;115(05):1100-1108

9 Jagarlamudi R, Kumar L, Kochupillai V, Kapil A, Banerjee U, Thulkar S. Infections in acute leukemia: an analysis of 240 febrile episodes. Med Oncol 2000;17(02):111-116

10 Zembower TR. Epidemiology of infections in cancer patients. Cancer Treat Res 2014;161:43-89

11 Freifeld AG, Bow EJ, Sepkowitz KA, et al; Infectious Diseases Society of America. Clinical practice guideline for the use of antimicrobial agents in neutropenic patients with cancer: 2010 update by the Infectious Diseases Society of America. Clin Infect Dis 2011;52(04):e56-e93

12 Karanwal AB, Parikh BJ, Goswami P, Panchal HP, Parekh BB, Patel KB. Review of clinical profile and bacterial spectrum and sensitivity patterns of pathogens in febrile neutropenic patients in hematological malignancies: a retrospective analysis from a single center. Indian J Med Paediatr Oncol 2013;34(02):85-88

13 Morris PG, Hassan T, McNamara M, et al. Emergence of MRSA in positive blood cultures from patients with febrile neutropenia-a cause for concern. Support Care Cancer 2008;16(09):1085-1088

14 Klastersky J, de Naurois J, Rolston K, et al; ESMO Guidelines Committee. Management of febrile neutropaenia: ESMO clinical practice guidelines. Ann Oncol 2016;27(Suppl 5):v111-v118

15 Weinstein MP, Lewis JS II. The Clinical and Laboratory Standards Institute Subcommittee on Antimicrobial Susceptibility Testing: background, organization, functions, and processes. J Clin Microbiol 2020;58(03):e1864-e19

16 Hakim H, Flynn PM, Knapp KM, Srivastava DK, Gaur AH. Etiology and clinical course of febrile neutropenia in children with cancer.J Pediatr Hematol Oncol 2009;31(09):623-629

17 Kar YD, Özdemir ZC, Bör ÖTurkish Archives of Pediatrics. Evaluation of febrile neutropenic attacks of pediatric hematologyoncology patients. Turk Pediatri Ars 2017;52(04):213-220

18 Rolston KV. Infections in cancer patients with solid tumors: a review. Infect Dis Ther 2017;6(01):69-83

19 Singh R, Jain S, Chabbra R, Naithani R, Upadhyay A, Walia M. Characterization and anti-microbial susceptibility of bacterial isolates: Experience from a tertiary care cancer center in Delhi. Indian J Cancer 2014;51(04):477-480

20 Klastersky J. Management of fever in neutropenic patients with different risks of complications. Clin Infect Dis 2004;39(Suppl 1): S32-S37

21 Yadegarynia D, Tarrand J, Raad I, Rolston K. Current spectrum of bacterial infections in patients with cancer. Clin Infect Dis 2003; 37(08):1144-1145

22 Kapoor G, Sachdeva N, Jain S. Epidemiology of bacterial isolates among pediatric cancer patients from a tertiary care oncology center in North India. Indian J Cancer 2014;51(04):420-424

23 Siddaiahgari S, Manikyam A, Kumar KA, Rauthan A, Ayyar R. Spectrum of systemic bacterial infections during febrile neutropenia in pediatric oncology patients in tertiary care pediatric center. Indian J Cancer 2014;51(04):403-405

24 Wisplinghoff $\mathrm{H}$, Seifert H, Wenzel RP, Edmond MB. Current trends in the epidemiology of nosocomial bloodstream infections in patients with hematological malignancies and solid neoplasms in hospitals in the United States. Clin Infect Dis 2003;36(09): 1103-1110

25 Tohamy ST, Aboshanab KM, El-Mahallawy HA, El-Ansary MR, Afifi SS. Prevalence of multidrug-resistant gram-negative pathogens isolated from febrile neutropenic cancer patients with bloodstream infections in Egypt and new synergistic antibiotic combinations. Infect Drug Resist 2018;11:791-803

26 Martinez-Nadal G, Puerta-Alcalde P, Gudiol C, et al. Inappropriate empirical antibiotic treatment in high-risk neutropenic patients 
546 Blood Stream Infections in Children with Chemotherapy-induced Febrile Neutropenia Sampagar et al.

with bacteremia in the era of multidrug resistance. Clin Infect Dis 2020;70(06):1068-1074

27 Talukdar A, Barman R, Sarma A, et al. Bacteriological profile and antibiotic sensitivity pattern of clinical isolates in a tertiary cancer care center in the northeast India. South Asian J Cancer 2020;9(02):115-119

28 Reddy R, Pathania S, Kapil A, Bakhshi S. Review of spectrum and sensitivity of bacterial bloodstream isolates in children with malignancy: a retrospective analysis from a single center. Indian J Cancer 2014;51(04):425-427

29 Garg VK, Mishra S, Gupta N, et al. Microbial and antibiotic susceptibility profile among isolates of clinical samples of cancer patients admitted in the intensive care unit at regional tertiary care cancer center: a retrospective observational study. Indian J Crit Care Med 2019;23(02):67-72 